\title{
DESIGN LAYOUT OF AN ISOBAR SEPARATOR BASED ON 5TH ORDER CALCULATIONS*
}

\author{
M. Portillo, J.A. Nolen, T.A. Barlow \\ Physics Division, ANL, 9700 S. Cass Ave., Argonne, IL 60439, USA
}

\begin{abstract}
A scheme for carrying out numerical simulation of a multistage mass separator is described. The results of a calculated solution are summarized for the design of a high resolution separator for purifying beams at the isobaric level.
\end{abstract}

\section{INTRODUCTION}

Success of nuclear experiments carried out at energies at or above the coulomb barrier depends largely on having a minimal amount of contamination by species of nuclei differing from the one of interest. For accelerating ions of radioactive nuclei the task of doing so can be especially challenging, since the source of ions is limited in intensity. It is necessary to use a mass filtering process that provides high purity beams while preserving transmission. For most cases a resolution of about $m / \Delta m=20,000$ is adequate at mass $\mathrm{A}=100$ to obtain a separation between isobars of mass excess difference of 5 $\mathrm{MeV}$.

We describe here the design for a device capable of purifying beams at the isobaric level and the simulation results from $5^{\text {th }}$ order calculations. A transverse emittance acceptance of $10 \pi \mathrm{mm}-\mathrm{mr}$ for ions at $100 \mathrm{keV} / 250 \mathrm{amu}(1$ $\mathrm{mm}$ entrance slit width and $\pm 20 \mathrm{mr}$ maximum divergence) and $\pm 10 \mathrm{eV}$ energy spread is assumed.

\section{ACROMATIC MASS SEPARATORS}

When considering mass separators at the level of isobar mass differences it is necessary to weigh in the effect of the beam energy spread from the ion source. The voltage ripple from modern ion source power supplies can be suppressed to the $10^{-5}$ level; however, plasma type ion sources impose energy spreads on the order of $10 \mathrm{eV}$. Thus, considering an energy spread at the $10^{-4}$ level is necessary.

Since pure magnetic separators disperse the ions according to rigidity, then the mass and energy dispersion are equivalent; i.e.

$$
(x, \delta m)=(x, \delta K)
$$

The energy dispersion tends to impose a limit on the resolution between ions of different mass. Therefore, it is necessary to utilize a spectrometer with achromatic character. It's noted that we have adopted the notation in which $x$ is the displacement in the horizontal plane with the $z$ axis oriented in direction of the beam. The variable $\delta m$ is the fractional difference in mass, $\Delta m / m$, and $\delta K$ for the kinetic energy.

The principle of an achromatic mass separator can be understood in terms of the fist order transport map in matrix form. Consider the horizontal plane position variables, $x$ and $a$, in a subspace with the mass and energy variables, $\delta m$ and $\delta K$, respectively. The origin of the coordinate axes is the position of the reference particle lying along the optic axis. If we construct a position vector $(x, a, \delta m, \delta K)$, then the transfer map can be expressed as,

$$
\mathbf{T}=\left(\begin{array}{cccc}
(x, x) & (x, a) & (x, \delta K) & (x, \delta m) \\
(a, x) & (a, a) & (a, \delta K) & (a, \delta m) \\
(\delta K, x) & (\delta K, a) & (\delta K, \delta K) & (\delta K, \delta m) \\
(\delta m, x) & (\delta m, a) & (\delta m, \delta K) & (\delta m, \delta m)
\end{array}\right)
$$

which yields the final position vector when we take its product with the initial position vector. The elements are determined by the equations of motion for the given electromagnetic fields distributions imposed by the system of optical elements [1]. Considering this subspace is sufficient as long as mid-plane symmetry is preserved.

\subsection{Double-Focusing Spectrometer}

Consider, for example, the double-focusing mass separator [2] like the one illustrated in Figure 1. The map of the system can be evaluated as the matrix product of the magnetic and electrostatic sections' maps, $\mathbf{T}_{B}$ and $\mathbf{T}_{E}$, respectively, to yield

$$
\mathbf{T}=\mathbf{T}_{E} \cdot \mathbf{T}_{B}
$$

Imposing point-to-point horizontal focusing at $\mathrm{B}$ and $\mathrm{C}$

results in the energy dispersion term

$$
(x, \delta K)=(x, x)_{E}(x, \delta K)_{B}+(x, \delta K)_{E}
$$

Requiring that this term also vanish implies that rays of different energy will get refocused at $\mathrm{C}$ as illustrated by the figure.

Some technical difficulties make this scheme unfavorable. Small mutual vertical inclinations between the electrode surfaces tend to misalign the beam from the mid-plane causing so called "parallelogram-type" defocusing [3]. The effect is sometimes called "parallelogram-type" defocusing and can cause severe losses in resolving power [4] 


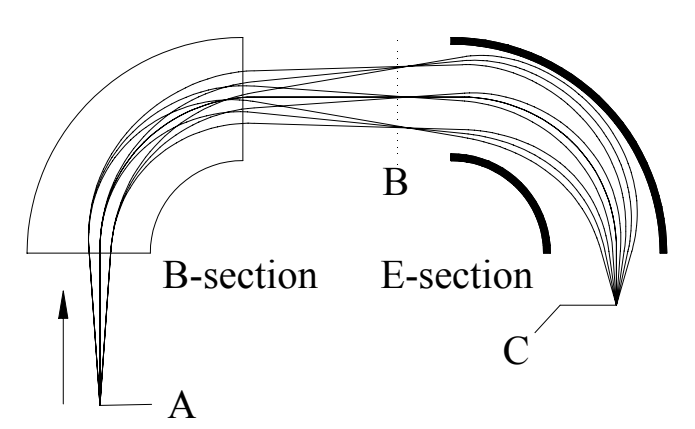

Figure 1. Double-focusing spectrometer with rays of multiple divergence and energy.

\subsection{Dual-Potential Spectrometer}

To avoid the effects of electrostatic condensers another method of energy focusing was devised which uses at least two stages of magnetic separation at different potentials [5]. Similar spectrometers have been devised in the past for eliminating unwanted scattered particles but not as achromatic spectrometers [6][7]. An achromatic system has been worked out here and the layout is illustrated in Figure 2.

The system is broken up into four sections. The beam enters through section $\mathrm{H}$ (points A-C) at $K_{H}=100 \mathrm{kV}$ then is decelerated through an immersion lens system in section I (from $\mathrm{C}$ to $\mathrm{D}$ ). The third stage (points D-F) lies at a $K_{L}=90 \mathrm{kV}$ potential on an isolated platform. The potential may vary as long as the ratio of the of the kinetic energies remains $K_{H} / K_{L}=10$. The radius of the dipoles in section $\mathrm{H}$ are $R_{H}=2.5 \mathrm{~m}$, while the radius of those in section L are just $R_{H} / \sqrt{10}$ in order to keep the same field strength for all the dipoles. Finally, the last section is simply the reverse of section I and accelerates the beam back to $K_{H}$ as the beam exits the isolated platform.

Magnetic multipole fields are imposed at the midpoint of both sections $\mathrm{H}$ and $\mathrm{L}$ (MH and ML3, respectively). The multipole $\mathrm{MH}$ provides quadrupole, hexapole, and octupole fields for correcting geometric and fringing field induced aberrations at section $\mathrm{H}$. Multipole ML3 contains up to duodecapole fields in order to correct to 5 th order the aberrations of section L, as well as the aberrations imposed by the immersion lenses. The remaining multipoles at section L (ML1, ML2, and ML4) are imposed for correcting higher order chromatic aberrations and also go up to 5th order.

The quadrupole fields keep maximize the $x$-beam envelope at the diopoles while allowing comparatively small oscillions in $y$ to maximize the resolving power [1]. The scheme also insures a minimum growth in $(x, b b)$ while the $(x, a a)$ is minimized by the hexapole correction.

For better beam stability, the fields were fit such that all four sections apply telescopic focusing on the beam. This means that the terms $(x, a),(a, x),(y, b)$, and $(b, y)$ all vanish simultaneously at $\mathrm{C}, \mathrm{D}, \mathrm{F}$, and $\mathrm{G}$.

The first order transfer maps for the first three sections can be evaluated and their matrix product,

$$
\mathbf{T}=\mathbf{T}_{L} \cdot \mathbf{T}_{I} \cdot \mathbf{T}_{H}
$$
gives the transfer map from point $\mathrm{A}$ to point $\mathrm{F}$. The resulting energy and mass dispersion terms are

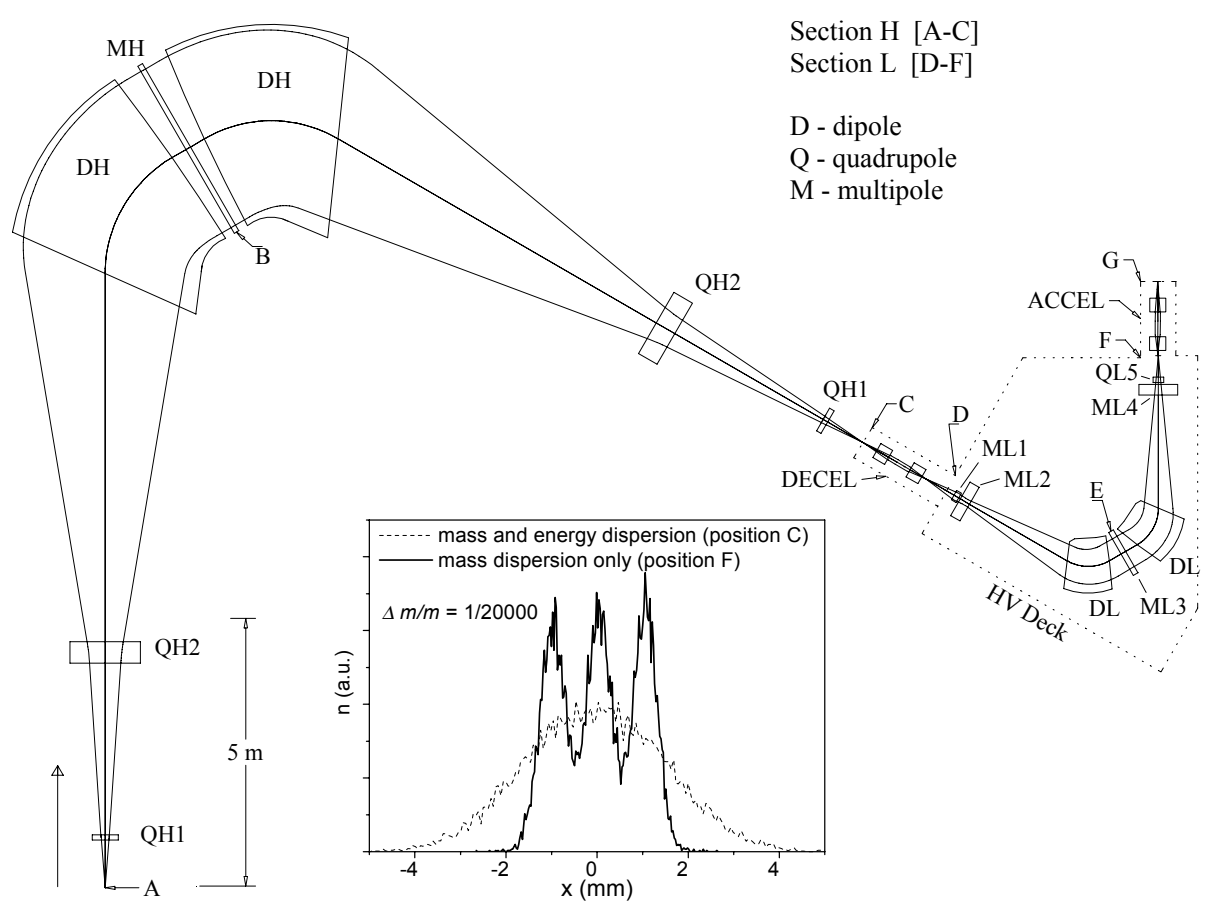

Figure 2. Layout of dual-potential spectrometer. The spectrometer can be broken up into 4 sections as described in the text. The inset shows a plot of the beam distribution at points $\mathrm{C}$ and $\mathrm{F}$. 


$$
(x, \delta K)=(x, \delta K)_{L}(\delta K, \delta K)_{I}-(x, x)_{I}(x, \delta K)_{H}
$$

and

$$
(x, \delta m)=(x, \delta m)_{L}-(x, x)_{I}(x, \delta m)_{H}
$$

respectively. The minus signs results since the symmetry of section $\mathrm{L}$ gives a magnification of $(x, x)_{L}=-1$. The fields and locations of the elements are optimized to make $(x, \delta K)$ vanish. By applying equation (1) to equation (6) under this condition we get that

$$
\frac{K_{L}}{K_{H}}=1 /(\delta K, \delta K)_{I}=\frac{(x, \delta m)_{L}}{(x, \delta m)_{H}(x, x)_{I}}
$$

Combining this with equation (7) we get that the ratio of the mass separation at the exit of section $\mathrm{H}$ to separation at the exit of section $\mathrm{L}$ is given by

$$
\frac{(x, \delta m)_{H}(x, x)}{(x, \delta m)(x, x)_{H}}=1-\frac{K_{L}}{K_{H}}
$$

This factor implies that there is a penalty in mass separation and reduces as $K_{L}$ becomes small compared to $K_{H}$. Unfortunately, technical problems arise from raising $\mathrm{KH}$ and the emittance grows at deceleration, thus setting limits on how small the ratio may be. Our choice of parameters implies that we should expect about a $10 \%$ loss in mass separation. This factor becomes negligible in terms of final mass resolving power obtain by factoring out the energy spread effects.

\section{NUMERICAL CALCULATIONS}

The COSY Infinity code system was used for calculation the beam optics. It can be used to evaluate arbitrary order maps and is equipped with efficient fitting algorithms [7][8]. A version of the code was modified to calculate maps of elements with DC acceleration and time-varying electromagnetic fields [9]. This was necessary for simulating the effect of the immersion lens systems. The transfer maps were carried out to 5 th order to determine the optimum parameters of the elements and correct the aberrations with the multipoles that were imposed. The corrections were carried out by minimizing the weighted second moments in transverse phase space.

The beam enters the spectrometer with an aspect ratio of $y_{m} / x_{m}=8$ so that the beam has a $1 \mathrm{~mm}$ full width along the $x$-axis and divergence of $a_{m}= \pm 20 \mathrm{mr}$. Such beams are obtainable by the use of quadrupole multiplets prior to the object slits as described in the literature [10].

The resulting beam envelope dimensions are plotted in Figure 3 for the 5 th order map calculation. The limits are determined by finding the statistical maxima at the $\pm x$ and $\pm y$ side of the optic axis when applying a Monte Carlo generated phase space distribution with sharp boundaries.

Finally, we apply a more realistic model for the phase space distributions by applying Gaussian like probability densities to the Monte Carlo generated phase space. The boundaries are set at 4 sigma so that $96 \%$ of the particles are lying within the set boundaries. The resulting distributions are plotted on the inset of Figure 2 for a mass difference of $\Delta m / m=1 / 20000$. For two adjacent peaks of equal intensity we expect a cross contamination of about $5 \%$ for a transmission efficiency of $96 \%$. This compares

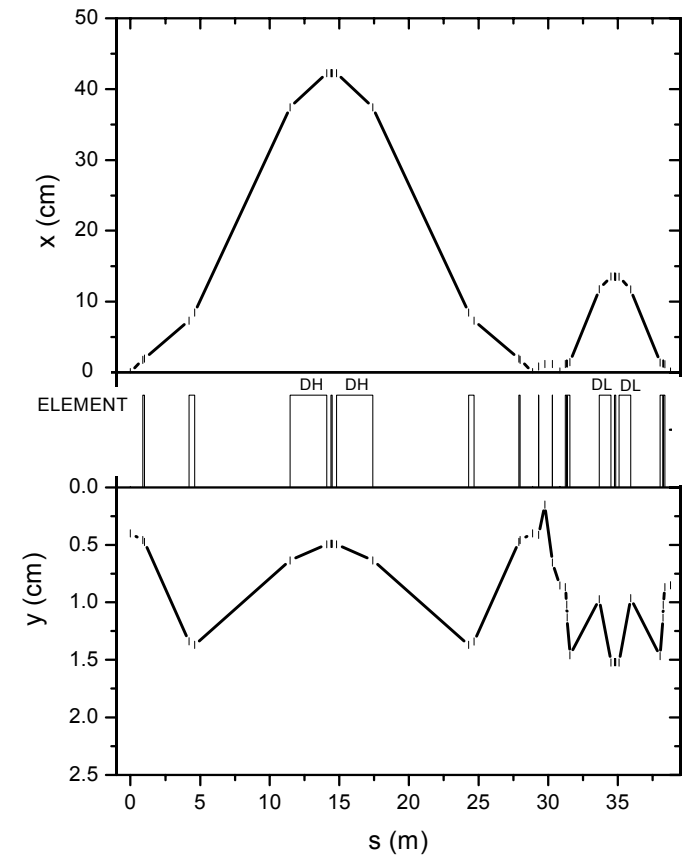

Figure 3. Beam envelopes for the dualpotential spectrometer in the horizontal and vertical planes as determined by 5th order

to a $1.5 \%$ cross contamination when applying only first order calculations.

The homogeneity of the field distributions in the dipoles is critical. There will be a need for trim coils in the dipoles to superimpose fields that suppress those caused by small ferromagnetic saturation effects. These types of coils are relatively trivial to insert at the pole tips and have been successfully used before [11].

\section{REFERENCES}

[1] H. Wollnik, Optics of Charged Particles, Academic Press, Orlando, FL (1987).

[2] M.I. Yavor, Nucl. Inst. Meth. B126 (1997) p. 266.

[3] M.I. Yavor and A.S. Berdnikov, Int. J. Mass. Spectr. Ion Proc. 128 (1993) p. 149.

[4] H. Matsuda, T. Matsuo and N. Takahashi, Int. J. Mass Spectr. Ion Phys. 25 (1977) p. 229.

[5] G.Ciavola, D. Rifuggiato, H. Weick, M. Winkler, H. Wollnik, Nucl. Inst. Meth. B126 (1997) p. 17.

[6] H. Wollnik, Nucl. Instr. Meth. A 363 (1995) p.393.

[7] M. Berz, Modern Methods in Particle Beam Optics, Academic Press, San Diego, 1999.

[8] COSY Infinity Web page and Manual. http://www.beamtheory.nscl.msu.edu/cosy

[9] A.A. Geraci, T.A. Barlow, M. Portillo, J.A. Nolen, K.W. Shepard, K. Makino and M. Berz, Calculation of Rado-Frequency and Electrostatic Structures Using Map-Oriented Beam Optics (to be published).

[10] H. Wollnik, Nucl. Instr. Meth. B 56 (1991) p. 1096.

[11] H. Wollnik, Nucl. Instr. Meth. 103 (1972) p. 479. 